\title{
SAMPLING CONCRETE BY TOO SMALL ELEMENTS; WHAT SHOULD WE DO TO GET RELIABLE INFORMATION?
}

\author{
PIET STROEVEN AND ZHANQI GUO \\ Faculty of Civil Engineering and Geosciences, Delft University of Technology, Stevinweg 1, 2628-CN Delft, \\ The Netherlands \\ e-mail: p.stroeven@tudelft.nl \\ (Accepted July 7, 2008)
}

\begin{abstract}
Macroscopically heterogeneous materials like concrete are generally sampled by too small, i.e., subrepresentative elements that can be either of 2D (section images) or of 3D nature (specimens). Based on scientific notions, like stochastic heterogeneity and structure-sensitivity, which are at the very heart of materials science and stereology, the paper demonstrates biases in obtained information to be generally inevitable when derived from such sub-representative designs. Only reliable comparison studies can be performed under the condition that the linear size of samples and of minimum structural dimensions (resulting from observation resolution) are maintained as fixed proportions of the relevant representative area and/or volume elements. This is demonstrated by three case studies.
\end{abstract}

Keywords: concrete, representative element, stochastic heterogeneity, structure-sensitivity.

\section{INTRODUCTION}

It is at the very heart of materials sciences and stereology that representative information on characteristics of structure and of the properties relying on it can only be derived from representative samples. This involves requirements as to location, orientation and size of samples. Such samples can be 2D in nature (images), or involve 3D units as used in materials testing programs. It is common knowledge among stereologists that location and orientation should be associated with random selections out of all possible solutions (in 2D and 3D). Further, the use of so called vertical sections is promoted by stereologists to obtain in economic way 3D information on structure. Also special attention is paid to the complete multi-stage sampling design. With the economy of the (quantitative image analysis) experiment as starting point, this has resulted in the popular saying: "Do more, less well!" (Gundersen et al., 1988). Relatively underrated in stereology is the topic of size of samples. This is probably so, because quite commonly materials and tissues have a characteristic length scale of structure that is much smaller than sample size. This is definitely not so for the category of macroscopically heterogeneous materials in which concrete takes up a major position. For such materials, the size of the samples in proportion to the largest structural dimension (generally maximum grain size) should be a dominant factor in research, to be discussed in this paper.

It is considered obvious nowadays that material properties will depend on features of material structure. Still, the impetus in concrete technology for going with research into depth of materials structure was traditionally not overwhelming. This is certainly due to the relatively low material costs involved, however as well to the high safety factors in building codes for the design of engineering structures in which the material is employed. The latter leads on the one hand to an excess of material used in engineering structures, on the other hand to relatively low working stresses. This could camouflage for a long time the limited insight into complicated material structure and resulting material performance. The development during the past decades of more sophisticated new types of special concretes that had to fulfil higher demands, as well as the worldwide confrontation with serious durability problems in the concrete infrastructure have significantly enhanced number and depth of studies into structure and performance of cementitious materials. The requirements of sustainable development will inevitably lead on the longer run to requirements to limit the excess material, which will further stimulate such research efforts.

Of course, samples of relatively huge dimensions would be required in the case of macroscopically 
heterogeneous materials like concrete. Even for additive functionals or descriptors of material structure, and for the structure-insensitive properties relying on it, a requirement would be using samples for normal concrete of four to five times maximum grain size, so, 100 to $150 \mathrm{~mm}$ (Cook and Seddon, 1956). Configuration-sensitive descriptors of material structure, and the structure-sensitive properties relying on it, could ask for significantly larger samples, depending on the degree of sensitivity, of course. This could lead to linear dimensions of the order of $10^{3} \mathrm{~mm}$, offering not an attractive perspective for systematic research efforts. Sub-representative sampling is therefore common practice, even in the case of microscopical studies in the framework of a multiscale modelling concept. Still, systematic research into concrete technology is time-consuming and expensive. The number of contributions coming from physical discrete element modelling (DEM) is as a consequence significantly increasing; research costs can be dramatically reduced. The structure of the aggregate is in such approaches considered separately from that of the fresh and thereupon matured cementitious binder. Although attractive, the two structures are not independent (Stroeven and Guo, 2006), complicating data evaluation in so called multi-stage simulation approaches.

Concrete is a macroscopically heterogeneous material. As a result, sizes of representative area or volume elements are generally large, and may become quite huge for structure-sensitive properties. As a result, common practice is to use sub-representative elements, with consequences as to reliability of obtained data; an issue mostly ignored in concrete literature. The only field where it is recognized that sub-sampling will lead to biased results is fracture mechanics of concrete, though the bias in tensile strength is generally denoted as the size effect. Extensive testing results on samples of various sizes have provided quantitative insight into this size effect. However, each parameter of material performance, and each descriptor of material structure on which such performance parameters rely, would require similar research efforts for the assessment of the different size effects. This is obviously an extremely time-consuming and thus expensive procedure, so offers a highly unrealistic concept.

This paper therefore formulates the scientific requirements that render possible designing a reliable comparison study in the sub-representative sampling domain. Since we are mostly interested in relative effects on characteristics of material performance due to modifications in technological parameters (e.g., water to cement ratio, particle size distribution/sieve curve, workability), this will offer a practical, economic and reliable concept for pursuing technological progress. The impact of the theoretical concepts on concrete research is additionally demonstrated by means of three case studies on different technological problems. These case studies partly deal with original research in which the authors were involved. Systematic research into material structure has been conducted mainly by means of (physical) discrete element computer simulation (DECS). This approach will be explicitly discussed, since some commonly applied systems violate the material science concepts laid down in preceding sections.

\section{MATERIALS SCIENCE CONCEPTS}

\section{STOCHASTIC CONCEPT OF HETEROGENEITY}

Heterogeneity is the scatter (e.g., expressed by standard deviation) among values of a descriptive parameter obtained from similarly sized samples, which is reflected by the probability density function of the selected parameter. Heterogeneity of a geometric parameter (e.g., crack length), or of the material property governed by this parameter, will increase for smaller elements as shown in Fig. 1 ( $\mathrm{Hu}$ and Stroeven, 2005). But heterogeneity among similarly sized volume elements will also change as a result of differences in the samples' observed micro-structural level (e.g., minimum crack length) implemented by differences in microscope magnification or strain gauge length. At increasing specimen or image size, heterogeneity will decline in a specific descriptor of structure or of the property relying on it. When heterogeneity is finally reduced to an acceptable low level, the resulting (2D or 3D) element is defined as homogeneous and denoted as the representative area/volume element (RAE/RVE).

Globalization for engineering purposes of data derived from sub-representative samples yield biased estimates of structural features or of physico-mechanical properties, unless in the situation of a composition parameter or of the associated structure-insensitive property (i.e., density and mass, respectively). This is illustrated by Fig. 1 (left). Even Young's modulus has been experimentally shown slightly structure-sensitive (Hershey, 1954; Stroeven, 1973). Biases will be more dramatic, of course, for some fracture properties and the underlying damage evolution characteristics, for which the dependence on structural details will be significantly larger. Fortunately, this high structure- 
sensitivity of the crack initiation strength will decline upon further energy dissipation due to microcracking; cracks release the high residual stresses developed during maturation of the material. Hence, the structure-sensitivity of the tensile strength is already reduced. As to the fracture energy, $G_{f}$, a low structuresensitivity can be expected, as has been proven by finite element approaches to SPACE-generated model concretes (Stroeven et al., 2002). Also, major part of the dissipated energy concerns the opening of the major crack of which the tortuosity is predominantly governed by volume fraction of the aggregate (with zero configuration-sensitivity) and not by the details of the sieve curve (Stroeven, 2000). This supports the proposal as to the relatively small size of the RVE for $G_{f}$ given in the Final Report of RILEM TC QFS (2004).

Hence, representative, unbiased information on structure-sensitive (e.g., fracture-related) properties can be derived only from specimens of representative size for the very property at issue.

Otherwise, when working with sub-RVE samples, correlations should be established between all observations on independent descriptive parameters and those of the associated RVEs.

In summary: heterogeneity in the geometric (structural) parameter that is considered relevant for the property of interest is reduced to an acceptable low level for the RAE. The RAE is therefore defined as homogeneous for that particular structural (geometric) parameter. The same can be stated for the hetero- geneity in a property directly measured on samples of the material (Freudenthal, 1950; Holliday and Thackray, 1964; Stroeven, 1973). Thus, the degree of heterogeneity increases with a diminishing size ratio of sample and RVE/RAE (Hu and Stroeven, 2005).

A quite common misconception of the heterogeneity phenomenon is revealed by a study performed by Keeton on cement paste, mortar and concrete (Keeton, 1965). $2 \mathrm{~mm}$ thick photo-elastic coatings, glued on the surfaces of compressed specimens, reflected according to the author growing disorder in shear strain contours with increasing grain size under illumination by mono-chromatic light. Keeton erroneously associated this with heterogeneity as if dealing with a material property and classified the materials accordingly. Keeton's conclusion on increasing heterogeneity at larger grain sizes is incorrect, since strains were sampled by constant coating thickness, although the size of the RVE increased with grain size. This should inevitably lead to increased heterogeneity as a reflection of the sampling strategy. Hence, experiments only confirmed the validity of the theory of stochastic heterogeneity. Heterogeneity should have been found similar when paste, mortar and concrete specimens had been subjected to similar sampling sensitivity (not possible by photo-elastic coating analysis), so on the same level of their microstructures. Fig. 2 presents some of Keeton's results for mortar and concrete.
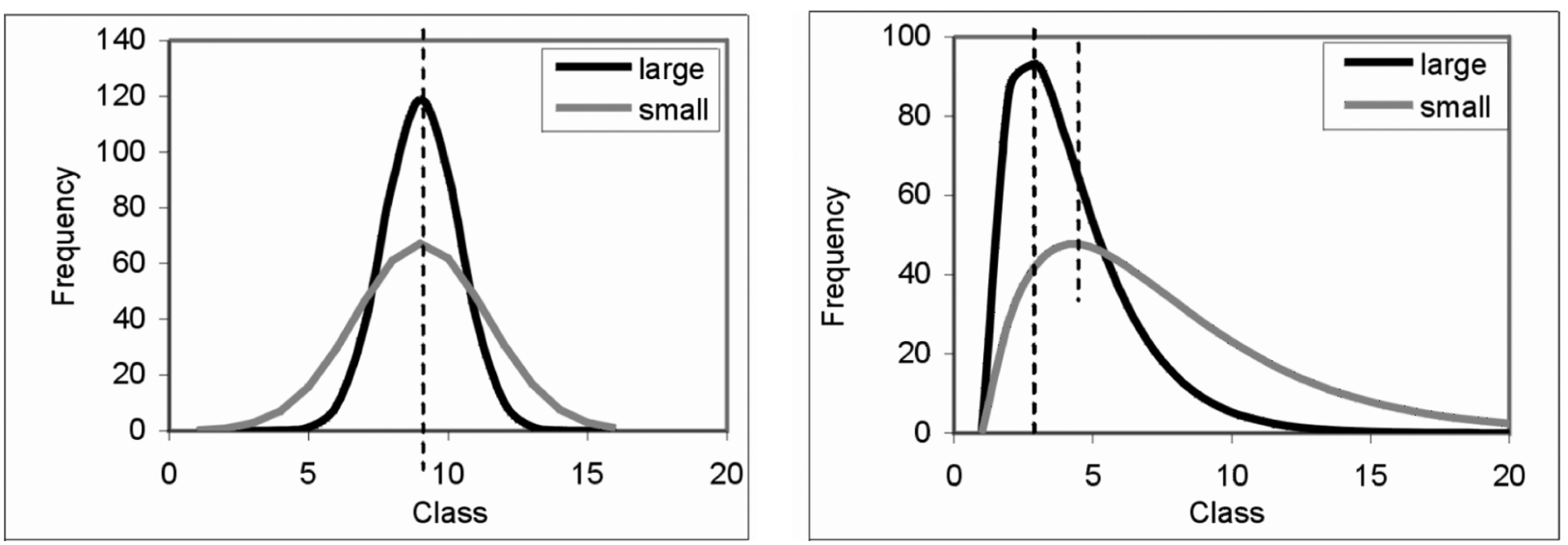

Fig. 1. Smoothed absolute frequency histograms of data on composition (left), and on configuration parameter (right) supposedly obtained from "large" and "small" elements (2D: field images; 3D: specimens) with respect to structural dimensions, say, maximum grain size. Number of fields is 400. Figure is reproduced from Stroeven and Guo (2006). 


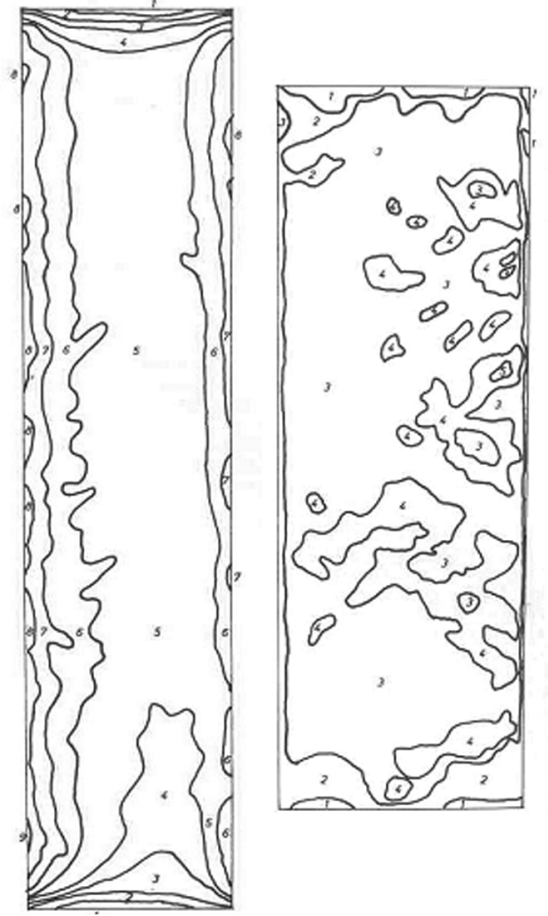

Fig. 2. Specimens of cementitious materials (mortar at the left and concrete at the right) covered by $2 \mathrm{~mm}$ photo-elastic coatings revealed under equal axial stresses in a standard photo-elastic set up isochromatics of which the chaotic character increased with increase in maximum grain size, and so with enlarged RVE (According to Keeton, 1965).

\section{SENSITIVITY TO MATERIAL STRUCTURE}

The shape of the probability density curve is symmetric (Gaussian) for a composition parameter or for a structure-insensitive property (Fig. 1, left). However, the curve will be asymmetric (skew) in the case of configuration- or of structure-sensitivity (Fig. 1, right) (Hu and Stroeven, 2005). Again, the width of the curve does not reflect the heterogeneity of the (geometric parameter, or property of the) material, but merely of the sampling scale. An experimental design based on constant sample (volume or area) sizes inevitably causes the sampling scale to be different for different structural parameters or material properties. For the same experimental design this would hold even for similar parameters or material properties (such as damage characteristics), but modified material structures, e.g., by increasing maximum grain size (Keeton's tests!), or by prolonged hydration (porosity).

Globalization of the data obtained on subRVE/RAE samples will not yield biases in engineering characteristics when dealing with composition homogeneity, or with the associated structure-insen- sitive property. However, this strategy fails in the case of non-zero values of the degree of configurationor structure-sensitivity. One is confronted with such conditions when investigating fracture properties and/ or the underlying geometric damage parameter(s). This is a highly relevant situation in materials research. Assessment of engineering properties on the basis of a sub-RVE sampling strategy requires therefore the availability of proper theoretical models, such as that of the weakest link on which Weibull's theory is based (Weibull, 1939). We will come back to this model in a case study presented later. With a lacking theoretical basis, correlations should be established experimentally between results from sub-RVE sampling designs and those obtained on relevant RVEs, conditions being similar, of course. And this has to be accomplished, basically, for each independent geometric parameter and associated material property, and for different environmental conditions; an extremely time-consumming and expensive job. Therefore, physical discrete element computer simulation (DECS) approaches can offer more economic alternatives, provided the real concrete ( $=$ realcrete) is reproduced sufficiently realistic. This will be discussed in what follows.

\section{COMPUTER SIMULATION APPROACHES}

Modelling pursues a schematization of reality with operational potentials. This indicates the intimate relationship between the type and degree of schematization and the objectives for the appeal on the operational potentials. We don't have to schematize all aspects of the inherently complex system that concrete definitely is, but only those relevant for obtaining this unbiased 3D structure information on which the aspect of material performance relies in which we are interested. A complicated task, anyhow. However, we might discover that at least some of our questions deal with aspects of material performance that have a low structure-sensitive nature. Hence, the schematization of reality is not very demanding for the simulation system when the researcher is targeting material density, or volume fractions of composing compounds of the material. These are composition characteristics of the material. The associated material properties that completely depend on composition are denoted as structure-insensitive properties (like mass, or approximately, Young's modulus). When investigating particle spacing, or local grading characteristics, one is dealing with configuration characteristics of the material. The associated material properties are referred to as structure-sensitive. So, the schematization of 
reality is quite demanding for the simulation system when the researcher's focus is on structure-sensitive properties. The computer-made concrete (compucrete) should be more sophisticated in such cases, because configuration of particles, the so-called group effect, will influence these properties.

Available physical computer simulation systems for packing of hard particles can be placed, therefore, in two distinctive groups. In the first, granular packing of spheres (or other idealized shapes) is realized by sequential random addition (SRA). The systems in the second group are based on so-called concurrent algorithms (CA) and pursue densification of a fixed number of particles. The strategy to solve the overlap problem in this group is either of static or of dynamic nature. In both cases, the container size is initially enlarged so that all particles can be positioned at Poisson points. Thereupon, the container size is gradually reduced and the particulate system squashed. The mechanical contraction in Williams and Philipse (2003) is a static solution, which involves local shifting of particle positions to eliminate overlap. The SPACE system that has been developed at Delft University of Technology realizes the compaction by a dynamic algorithm. SPACE is based on spherical particles only. However, presently a new concurrent system (HADES) is in use, allowing the simulation of arbitrarily shaped particles (Stroeven and Guo, 2006; Stroeven et al., 2006). This is to account for particle shape effects that have more serious impact on packing than so far assumed in concrete technology.

Compaction by vibration is employed for producing in practice dense concretes. This may stimulate size segregation of aggregate (Brazil Nut Effect: Fig. 3, see Stroeven, et al., 2007b), and of the cement grains in the so called interfacial transition zones (ITZs) around aggregate particles (Stroeven and Stroeven, 2003). The latter is crucial for obtaining dense and strong ITZs that constitute in normal concrete the weakest link in the mechanical system (Fig. 4). However, this phenomenon might also have paramount impact on morphology and topology of porosity, and thus on concrete durability (Stroeven and Guo, 2006). This and other configuration-sensitive features of particle packing are correctly reproduced by SPACE system (Stroeven et al., 2007a). Hence, the information coming from investigations of material structure and used for the case studies can be considered reliable.

\section{CASE STUDIES}

The impact the theoretical concepts of stochastic heterogeneity and structure-sensitivity will have on reliability of comparative studies will be elucidated in the forthcoming practical case studies featuring both realcrete and compucrete. The compucrete is produced by DECS system SPACE. The first case study deals with the composition parameter "porosity". Although basically approached experimentally, the complicated topological transformation process of pore depercolation during hydration is studied on model cement paste. The second case study assesses on model cement paste the configuration-sensitive property "particle spacing", governing to some degree various properties of the material. The last case study has very old roots, but is the sole field in concrete technology (engineering fracture mechanics) whereby a size effect is recognized in sub-representative testing. Research results used in the first two case studies have partly been published earlier in Image Anal Stereol, to which is explicitly referred.
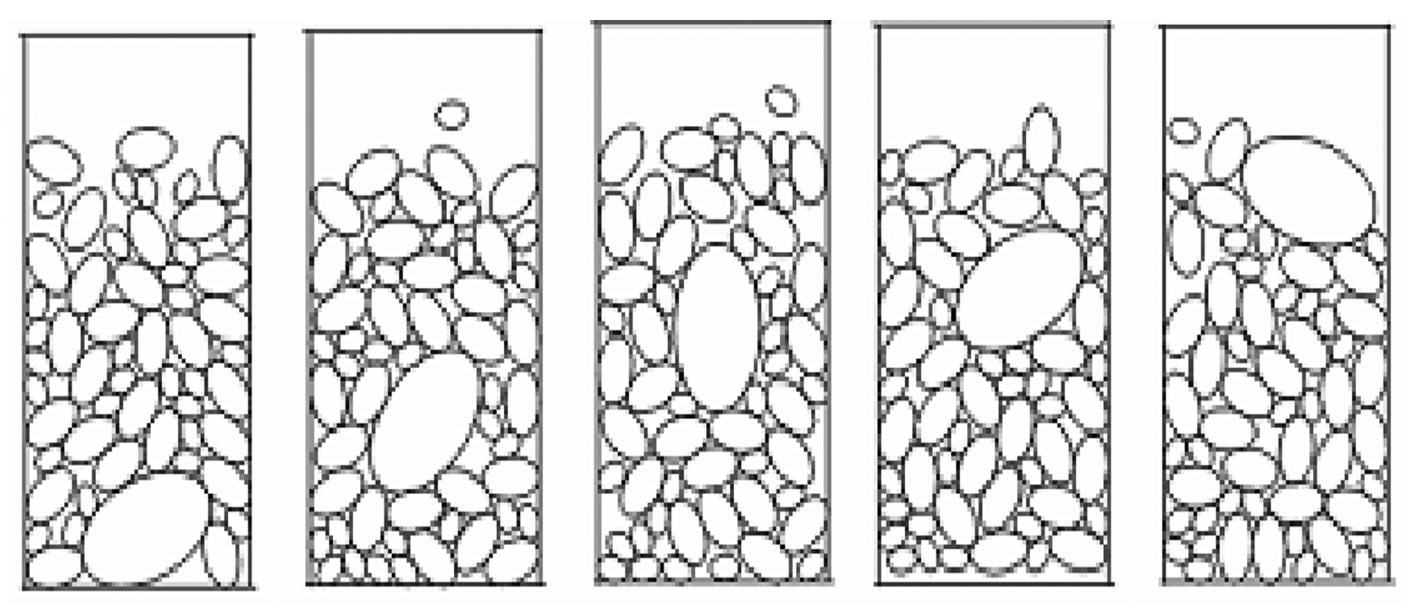

Fig. 3. Traditional Brazil nut concept: mixture of non-spherical particles of various sizes is activated by vibration at the bottom of the container. Sequence of $2 D$ simulations by HADES during the size segregation process is displayed (for video, see Stroeven et al., 2006). 


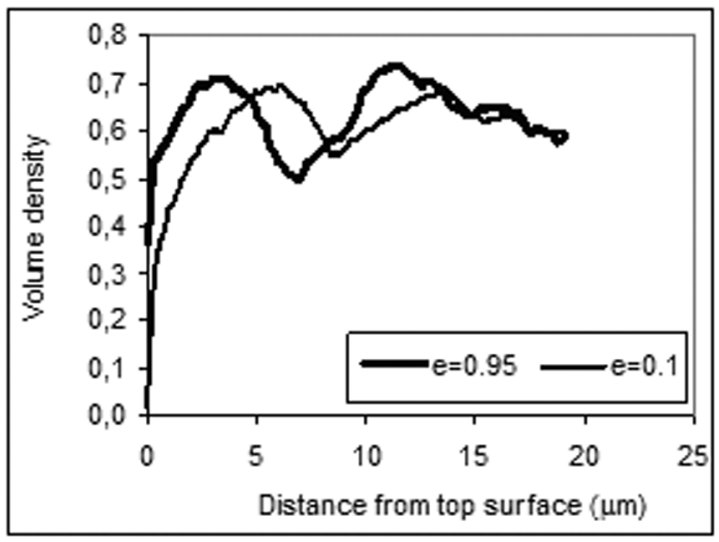

(a) Volume density of binder

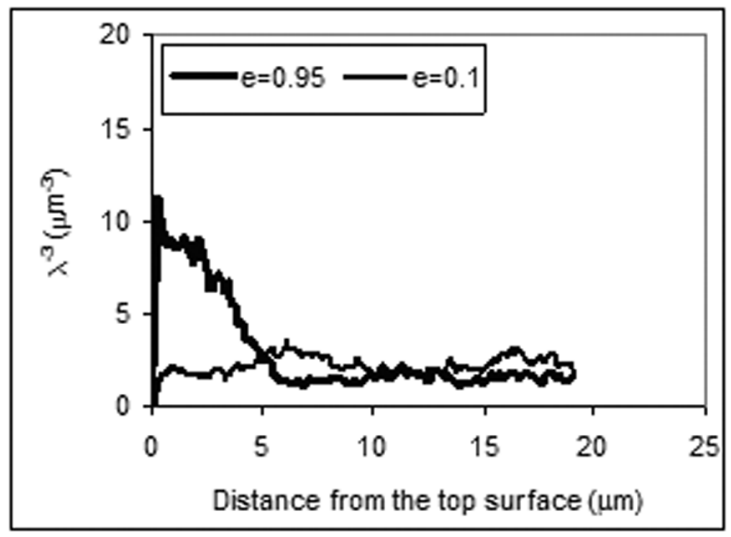

(b) Global bonding capacity in binder

Fig. 4. Effect of workability of fresh binder with discontinuous grading on density (a) and on internal bonding capacity (b) near aggregate surface (at the left). High value of e (=energy dissipation during compaction) corresponds to better workability. Physical (van der Waals) bonding is supposedly proportional to $\lambda^{-3}$, whereby $\lambda$ stands for the mean free spacing (Underwood, 1968). Note dramatic drop in $\lambda^{-3}$ due to incomplete size segregation (Brazil Nut Effect?) under compaction by vibration (Stroeven and Guo, 2006).

\section{Composition heterogeneity}

Volume fraction of pores (attributed as porosity) in hydrated cement paste is chosen as a composition parameter (with zero configuration-sensitivity) to explore composition heterogeneity at different structural levels (i.e., due to different sampling sensitivity levels) of cementitious materials (Chen et al., 2006). The forthcoming experimental approach could have been accomplished easily by SPACE-produced compucrete. Fig. 5 gives an example from a study on self-healing capacity of concrete due to anhydrate cement left after hydration (He et al., 2007). Local porosity distribution has been determined on similar section images on which fields of constant size were delineated by superimposing a square grid. Assessment of areal pore fraction was accomplished by point counting.

Based on local porosity theory (Hilfer, 1991), Hu and Stroeven (2005) experimentally studied in small elements of ordinary cement paste with various water cement $(\mathrm{w} / \mathrm{c})$ ratios the changes imposed by hydration on local porosity distribution. Section images of $263 \times 186 \mu \mathrm{m}$ were made by back-scattered electron microscopy at optical resolution of $0.18 \mu \mathrm{m} /$ pixel. These images were sub-divided into fields of constant size of which porosity was determined, allowing construction of the probability density curve. The shape of this curve depends not only on technical parameters, but also on field size. Typical differences between the curves are described in $\mathrm{Hu}$ and Stroeven (2005). Heterogeneity of porosity in the cement paste is seemingly increasing during the hydration process, when based on fields of similar size. This conclusion is incorrect because observations and comparisons were made on different structural levels. Cement hydration gradually changes pore space from a connected structure to a de-percolated network with some isolated pores, yielding a significant decline in the number of pore features in the image plane. In the viewpoint of statistics, the smaller number of pore features leads to larger scatter of porosity data. This larger scatter is seemingly due to increased heterogeneity of porosity. In fact, the correct interpretation should be that it points toward hydration-driven increasing linear dimensions of the RAEs for porosity.

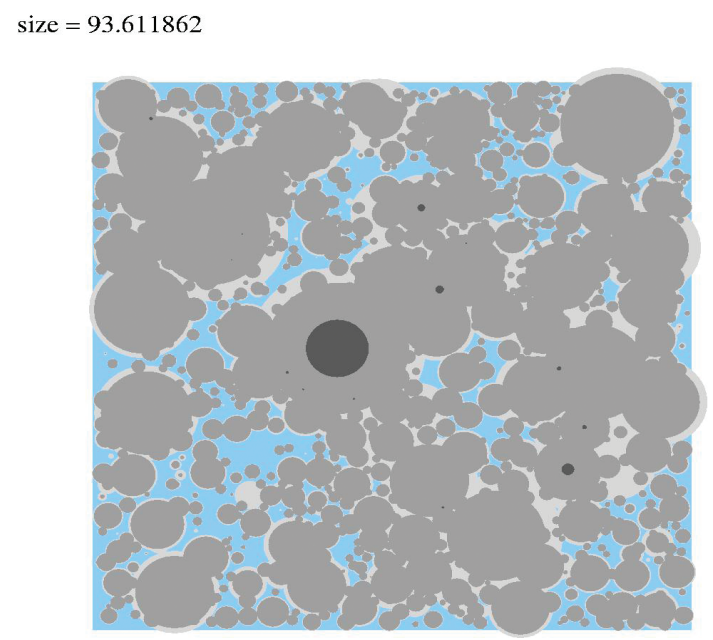

Fig. 5. Cube section of 1-year old cement paste with $w / c=0.5$ (gel in grey, air in light pink, water in blue, unhydrated cement in black). Section is covered by square grid of straight lines delineating fields in which values of local porosity are determined. 
Field sizes should be modified to the same proportion of the respective RAEs for a proper assessment of heterogeneity of porosity in cement pastes hydrated to different extents. This renders possible comparing outcomes on the same level of aggregation. This issue of 'continuous scaling' is explicitly discussed in Stroeven (2003). When the linear dimension of the RAE, $L_{\mathrm{RAE}}$, exceeds the image size as in this study, the same 'level of microstructure' (i.e., the same coefficient of variation in the geometric parameter at issue) can be achieved by taking the ratio of field size $L$ to $L_{\mathrm{RAE}}$ constant for all investigated specimens. These specific values of field size are denoted as $L^{*}$ (Hu and Stroeven, 2005). When the measurements are based on the same level of microstructure for the three cement pastes (at the appropriate values of $L^{*}$ ), the $\mu$ curves (normalized by their respective peak values) resemble each other quite close (Hu and Stroeven, 2005). Theoretically, the three $\mu$ curves should be identical and conform to the normal distribution after eliminating the aging effect (by shifting the curves, so that the peak values of the three curves coincide). The actual deviations of the experimental data from the normal distribution function were assessed by means of a $\chi^{2}$-test. For this purpose, the local porosity data are collected into traditional histograms. Details of the $\chi^{2}$-test and the obtained results can be found in $\mathrm{Hu}$ and Stroeven (2005). They show that at the $90 \%$ confidence level, the hypothesis can be accepted that in all three pastes porosities were distributed according to the same normal distribution curve. So, results confirm the concept of stochastic heterogeneity. These observations were based on images made with the same optical resolution. If the measurement sensitivity (resolution) had been also adjusted to the size of the RAE, the peakedness of the curves would have been increased, causing the curves to match even better.

This case study allows drawing the following conclusions:

a) The probability density curves are Gaussian, confirming porosity to be structure-insensitive.

b) Heterogeneity in porosity is the same in a quantitative image analysis approach to an hydrating system when the linear size ratio of sample and RAE is kept constant, and

c) when the measuring sensitivity is adjusted to changes in the size of RAE.

\section{Spacing heterogeneity}

The second case study deals with a particle spacing parameter of moderately high degree of configuration sensitivity, i.e., surface-to-surface spacing between nearest neighbors. This spacing parameter is denoted as $\Delta_{3 \mathrm{~s}}$ in three-dimensional space, and $\Delta_{2 \mathrm{~s}}$ in a section plane of the material body. Although the center-tocenter nearest neighbor spacing, $\Delta_{3}$, is defined in stereological literature (Underwood, 1968), the surfaceto-surface spacing may be more relevant for studying certain material properties. $\Delta_{3 \mathrm{~s}}$ has been used earlier for studying the spatial dispersion of aggregate grains in concretes (Hu, et al., 2005). The surface-to-surface spacing may govern physical (van der Waals-type) contributions in cementitious binder systems to strength, which have been demonstrated quite significant at lower water to cement ratios (Detwiler and Mehta, 1989; Goldman and Bentur, 1993; Bui et al., 2005). The surface-to-surface spacing of aggregate grains in combination with relevant information on extent of the interfacial transition zone (ITZ) can be used in modeling damage evolution in concrete (Stroeven et al., 2002). A case study will therefore be presented dealing with this highly relevant geometric feature. It will serve to demonstrate the effects of sampling strategy in a case of sensitivity to details in the material structure.

This issue is approached by computer simulation with the SPACE system. Three aggregate structures of model concrete were generated with constant volume fraction of aggregate $(50 \%)$. The aggregates conform in all cases to a continuous size distribution with a ratio of maximum to minimum grain size of two (i.e., diameters ranging from 5 to $10 \mathrm{~mm}$ ). The linear dimensions of the reference frame (cubic container) were $253 \mathrm{~mm}, 92.6 \mathrm{~mm}$ and $46.3 \mathrm{~mm}$, respectively, representing different sampling scales. By using periodic boundary conditions for the container, the volume element will represents bulk material (no boundary disturbances). The dispersion of the aggregate grains in mixtures is characterized by the distribution probabilities of the spacing parameter $\Delta_{3 s}$, denoted as $f\left(\Delta_{3 s}\right)$. The surface-to-surface distances between all nearest neighbours were determined inside the reference frame and thereupon classified according to their length to obtain the probability density curve. The probability density curves reveal differences with respect to "degree of heterogeneity" and "sample averages" as a function of the sampling scale (Fig. 6). Table 1 presents the mean and mode values of the spacing parameter $\Delta_{3 \mathrm{~s}}$. 

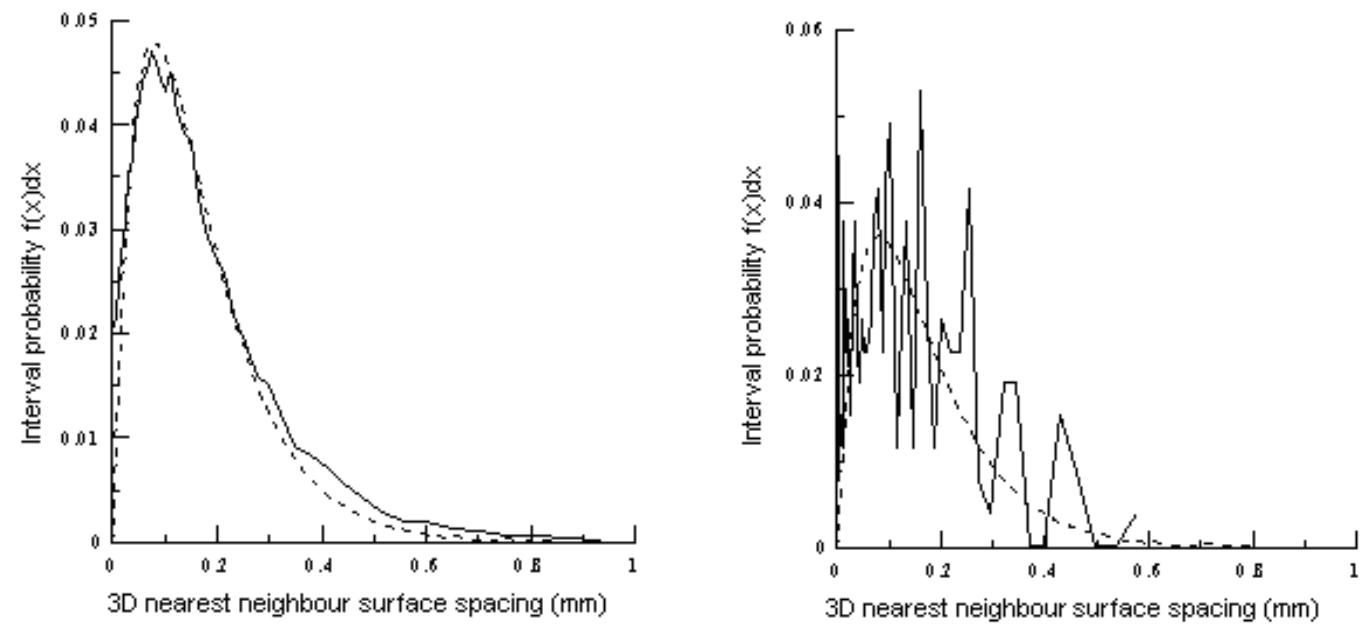

Fig. 6. Probability density functions of nearest neighbour surface spacing of same particle mixture (5-10 mm continuous grading) in largest (left) and smallest container (right). Continuous line: DECS results; dashed line: approximation by exponential function.

Table 1. Averages pertaining to different sampling levels of surface-to-surface distances $\Delta_{3 s}$ among neighbouring grains in the same aggregate of 5 to 10 mm grains (all dimensions in $\mathrm{mm}$ ).

\begin{tabular}{ccc}
\hline Container size & Mean spacing & Mode spacing \\
\hline 253 & 0.1083 & 0.0751 \\
92.6 & 0.1062 & 0.0993 \\
46.3 & 0.0989 & 0.1640 \\
\hline
\end{tabular}

The experimental curves were approximated per specimen size by:

$$
y=\mathrm{ax} e^{-\mathrm{b} x}
$$

The values of $a$ and $b$ in Eq. 1 were determined by regression analysis. The experimental distribution curves of the surface spacing and the fitting curves according to Eq. 1 are shown in Fig. 6 for the two extreme cases. Clearly, the probability density functions for $\Delta_{3 s}$ are skew, with a concentration of relatively small values of the nearest neighbour surface-tosurface distance. This is in agreement with theoretical predictions (Kendall and Moran, 1963). The rule of thumb estimate for composition homogeneity is 4 5 times the largest structural dimension (Cook and Seddon, 1956), i.e., $40 \sim 50 \mathrm{~mm} . \chi^{2}$-values derived from data presented in Fig. 6 revealed the linear dimension of the RAE to be approximately $300 \mathrm{~mm}$, exceeding to a considerable degree the estimate for composition homogeneity (Stroeven and Stroeven, 2001). This is consistent with predictions of Brown (1965) for composition and configuration homogeneity of conglomerates, and experimental observations on aggregate spacing by Stroeven (1973).
This case study allows drawing the following conclusions:

a) The probability density functions $f\left(\Delta_{3 s}\right)$ are strongly skewed to the left (Fig. 6). Surface-to-surface spacing is a configuration-sensitive geometric parameter, indeed;

b) Serious biases result when mean or mode values of representative elements (for engineering purposes!) are derived from sub-representative sampling designs (Table 1); this is analogous to so-called 'size effect' in fracture mechanics testing;

c) Heterogeneity in the geometric parameter at issue increases considerably for the same material when sample size is reduced with respect to RAE size (Fig. 6).

d) Stochastic heterogeneity effects in 3D nearest neighbour surface spacing will be excluded in a comparative study when the aforementioned size ratio is kept constant.

\section{Brittle strength heterogeneity}

The interest in the stochastic background of weakest link problems goes back about one century (Pearson, 1902), and major statistical contributions to estimating fracture properties, recognizing their structure-sensitive background, appeared during the $1920^{\text {th }}$ to $1940^{\text {th }}$. Analyzing the opinions at that time, Epstein (1948a) concluded "Many scientists accept the fact that the Gaussian distribution plays a fundamental role in science and, in fact, there are many who feel that this is the only distribution which 
nature calls truly her own ... however, in a certain class of phenomena the characteristic distributions are far from normal and are, in fact, strongly skewed to the left". Although this seems more widely accepted nowadays in engineering approaches to fracture problems, the underlying heterogeneity concept is still widely ignored, and the implications missed. That is the very reason to take an case with such old roots; what is presented is therefore far from new, but by many somehow overlooked or at least underestimated as to its consequences. Therefore, the case will also be presented in its "classical" form.

It is commonly assumed in case of concrete that flaws in the material body are at the basis of fracture properties. For the present - illustrative - purpose, the crack initiation (or brittle) strength is selected as a property highly sensitive to material structure. Nowadays, flaws are associated in normal concrete with more porous areas in the Interfacial Transition Zones (ITZ) around aggregate particles. Weibull (1939) has elaborated a theoretical concept for brittle fracture based on the weakest link concept for elementary units containing single flaws. The probability density function of the brittle strength of sub-volumes of the material body containing a single elementary flaw (here referred to as elementary units) is denoted by $f(\sigma)$, and the cumulative frequency curve by

$$
F(\sigma)=\int_{-\infty}^{\sigma} f(t) d t
$$

When the material body encompasses a total number of $i$ elementary units, the probability density function $g_{i}(\sigma)$ of the minimum value of brittle strength is given by (Cramér, 1946; Gumbel, 1960)

$$
g_{i}(\sigma)=i . f(\sigma)[1-F(\sigma)]^{i-1},
$$

with the cumulative distribution function $G_{i}(\sigma)$

$$
G_{i}(\sigma)=\int_{-\infty}^{\sigma} g_{i}(t) d t=1-[1-F(\sigma)]^{i}
$$

Determination of the median value of brittle strength, $\tilde{\sigma}$, is straightforward (Pearson, 1902). The mode, $\hat{\sigma}$, is obtained upon differentiation of Eq. 4 and equating to zero. The resulting equation cannot be solved in an elementary way for normally distributed brittle strength values of the elementary units. A new variable $z$ is therefore introduced defined by $z=i . F(\sigma)$. The cumulative and probability density functions of $z$ can be obtained for the limiting case of $i \rightarrow \infty$

$$
G_{i \rightarrow \infty}(z)=1-e^{-z} \text { and } g_{i \rightarrow \infty}(z)=e^{-z} .
$$

An asymptotic development allows expressing $\sigma$ into $z$ (Tippet, 1925; Epstein, 1948b)

$$
\sigma=m-s\left[\sqrt{2 \ln i}-\frac{\ln \ln i+\ln 4 \pi}{2 \sqrt{2 \ln i}}-\frac{\ln z}{\sqrt{2 \ln i}}\right],
$$

in which $m$ and $s$ are the mean and standard deviation of the brittle strength of the elementary units, governed by the normal distribution function $f(\sigma)$. The most probable value (mode) and variance of $\sigma$ are given respectively by

$$
E(\sigma)=m-s\left[\sqrt{2 \ln i}-\frac{\ln \ln i+\ln 4 \pi}{2 \sqrt{2 \ln i}}\right]
$$

and

$$
D^{2}(\sigma)=\frac{\pi^{2} s^{2}}{12 \ln i}
$$

The mean and standard deviation of the material containing a very large number of flaws can be formulated by $\hat{\sigma}=m-\alpha_{i} s$ and $s_{i}=\beta_{i} s$ in which the coefficients $\alpha_{i}$ and $\beta_{i}$ are given by

$$
\alpha_{i}=\sqrt{2 \ln i}-\frac{\ln \ln i+\ln 4 \pi}{2 \sqrt{2 \ln i}}
$$

and

$$
\beta_{i}=\frac{\pi}{2 \sqrt{3 \ln i}}
$$

Tables are available in the literature for $\alpha_{i}$ and $\beta_{i}$ and (Pearson, 1924; 1931). The decline rate in scatter with increasing sample volume is exceeding the one in strength. So, also the coefficient of variation is declining with increasing sample volume. In other words, sampling sub-volumes of the RVE (defined at an acceptable level of scatter) will lead to improved strength values accompanied by disproportionately increased scatter (or, heterogeneity). The strength ratio of an arbitrary material volume and the RVE is obtained by means of Eqs. 7 and 8, yielding

$$
\frac{\bar{\sigma}}{\bar{\sigma}_{R V E}}=\frac{m-\alpha_{i} s}{m-\alpha_{R V E} S} \approx 1-\frac{s}{m}\left(\alpha_{i}-\alpha_{R V E}\right)
$$

with

$$
\alpha_{i}-\alpha_{R V E}=\left(\frac{\beta_{R V E}}{\beta_{i}}-1\right) \alpha_{R V E}
$$


Hence, the strength increase due to sub-RVE sampling strategy is reflected by

$$
\frac{\bar{\sigma}}{\sigma_{R V E}} \approx 1+\frac{s}{m}\left(1-\frac{\beta_{R V E}}{\beta_{i}}\right) \alpha_{R V E} .
$$

Eq. 11 depicts the strength increase by stochastic heterogeneity on sub-RVE sampling level. The value of $\beta_{R V E}$ indicates the acceptable scatter limit $\left(=s_{i} / s\right)$ for declaring the sample volume representative for brittle strength, and thereby defining it as homogeneous. Heterogeneity is expressed by the ratio $\beta_{i} / \beta_{R V E}=s_{i} / s_{R V E}$. The microscopic material parameter $(\mathrm{s} / \mathrm{m})^{2}=v$ has been referred to in the international literature as the unit coefficient of variation (Tucker, 1927). When $\beta_{R V E}$ is selected, $\alpha_{R V E}$ is given by the relationship $\alpha_{R V E}=\pi /\left(\sqrt{6} \beta_{R V E}\right)$. Upon combination of Eqs 8 and 11, the ratio of linear dimension of sample and the RVE (proportional to $\sqrt[3]{i / i_{R V E}}$ ) is introduced as the running parameter. Hence

$$
\frac{\bar{\sigma}}{\sigma_{R V E}} \approx 1+\frac{s}{m} \alpha_{R V E}\left(1-\frac{\sqrt[3]{\ln i}}{\sqrt[3]{\ln i_{R V E}}}\right) .
$$

This defines the so-called size effect (RILEM TC QFS, 2004), in the present case supposedly for the crack initiation strength (Fig. 7). The unit coefficient of variation in Eqs. 11 and 12 is available for adjusting to experimental data. This paper is not intended, however, to discuss statistical strength theories.

The following conclusions can be draw from this third case study:

a) The probability density function of $\hat{\sigma}$ is strongly skewed to the left (Eq. 8); we deal with a performance parameter that is extremely structuresensitive. Moreover, ample evidences can be found in the literature on the skewness of the probability density function of concrete's tensile strength;

b) Serious biases in engineering strength parameters (mode, scatter) result from sub-RVE sampling designs; these are the so-called 'size effects';

c) Heterogeneity in the strength parameter increases disproportionately at reduced sample size, so also "coarseness" (coefficient of variation) increases ( $\beta i$ in Eq. 8);

d) Heterogeneity in the strength parameter (Eq. 8) is also influenced by the minimum crack length considered (governing $i$ in Eq. 8).

e) Stochastic heterogeneity effects on performance parameter (brittle strength) will be excluded in a comparative study when size ratios of specimen and of minimum crack length (resolution!) are kept constant with respect to linear dimension of relevant RVEs.

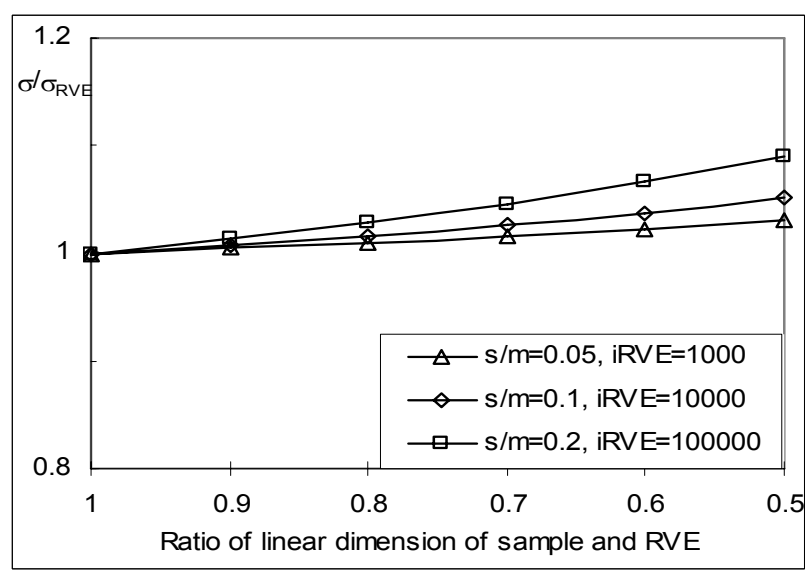

Fig. 7. Size effects for crack initiation strength according to Eq. 12 for combinations of different assumptions as to the unit coefficient of variation and the acceptation level of stochastic heterogeneity.

\section{DISCUSSION}

Heterogeneity is not a material characteristic. Instead, it is a stochastic concept that governs the design of experiments; it inevitably results from subrepresentative sampling procedures, either in 3D (material testing) or in 2D (image analysis) approaches. Homogeneity of a geometric parameter or of the associated material property can only be achieved for elements that are large enough to reduce the betweensamples scatter in the relevant parameter or property to an acceptable level. This holds for so-called Representative Volume Element as well as Representative Area Element (RVE/RAE). When the experimental design is based on volume (in engineering testing) or on area elements (in quantitative image analysis) of subrepresentative size as to the property or geometric feature of interest, the associated degree of heterogeneity is a function of the size ratio of sample and representative element.

Hence, biases will be similar in comparative studies on cementitious materials executed under equal conditions when this size ratio is maintained at constant level. This implies that sample size has to be adjusted to changes in the size of RVE or RAE due to material modifications (e.g., due to different maximum grain sizes in concrete, or to different maturities of cement paste). So, the requirement of constant size ratio of sample and RVE/RAE will generally require experimental designs encompassing different sample sizes. Globalized data on heterogeneous parameters (for 
engineering purposes) obtained in sub-represen-tative sampling designs are inevitably biased; this is leading to a parameter-related "size effect", as is gradually being recognized in fracture mechanics testing. In engineering testing (employing volume elements), as well as in materials investigations (based on area elements), all results should be corrected therefore to obtain unbiased engineering estimates. These correlations should be either obtained experi-mentally or analytically, based on models of the material in which heterogeneity is a continuous function of the size ratio of sample and RVA/RAE.

Geometric parameters can reflect to a certain degree material configuration. When this dependence is zero, the parameter is defined as a composition parameter (e.g., volume fraction). In the other case, we deal with a configuration-sensitive parameter that will depend on the group pattern of relevant particles (size, shape, dispersion). The same holds for the material property associated with certain features of material structure. These properties range between structure-insensitive (e.g., mass) and structure-sensitive (e.g., crack initiation strength). The size of the $\mathrm{RVE} / \mathrm{RAE}$ is a direct reflection of the degree of sensitivity to configuration of the geometric parameter, or to structure of the material property. This implies each geometric parameter and each material property to have its own independent scale of heterogeneity and at its very end the -by definition- homogeneous $\mathrm{RVE} / \mathrm{RAE}$ with dimensions specific for that parameter/ property.

\section{CONCLUSIONS}

Heterogeneity effects (so, biases) on global estimates in a comparative study in materials testing or in a comparative study based on quantitative image analysis can be excluded by maintaining the linear dimension of the samples (3D or 2D element), as well as of the smallest structural features taken into consideration (grains, pores, cracks) as fixed proportions of the size of the appropriate RVEs/RAEs. Only under such conditions can the experiments be analyzed in an unbiased way.

\section{REFERENCES}

Brown CB (1965). Minimum volumes to ensure homogeneity in certain conglomerates. J Franklin Inst; 279:189-99.

Bui DD, Hu J, Stroeven P (2005). Particle size effect on the strength of rice husk ash blended gap-graded Portland cement concrete. Cem Concr Comp 27(3):357-66.

Chen H, Stroeven P, Ye G, Stroeven M (2006). Influence of boundaery conditions on pore percolation in model cement paste. Key Engr Mat 303:486-92

Cook RW, Seddon AE (1956). The laboratory use of bonded-wire electrical resistance strain gauges on concrete at the Building Research Station. Mag Concr Res 8:31-8.

Cramér H (1946). Mathematical methods of statistics. New Jersey: Princeton University Press.

Detwiler RJ, Mehta PK (1989). Chemical and physical effects of silica fume on mechanical behaviour of concrete. ACI Mat J 86(6):609-14.

Epstein B (1948a). Statistical aspects of fracture problems. J Appl Phys 19:140-7.

Epstein B (1948b). Applications of the theory of extreme values in fracture problems. J Amer Statist Ass 43: $403-12$.

Freudenthal AM (1950). The inelastic behaviour of engineering materials and structures. New York: Wiley.

Goldman A, Bentur A (1993). The influence of microfillers on enhancement of concrete strength. Cem Concr Res 23:962-72.

Gumbel E (1960). Statistics of extremes. New York: Columbia University Press.

Gundersen HJG, Bendtsen TF, Korbo L, Marcussen N, Møller A, Nielsen K et al. (1988). Some new, simple and efficient stereological methods and their use in pathological research and diagnosis. Acta Path Microbiol Immunol Scandinavia 96:379-94.

He H, Guo Z, Stroeven P, Hu J, Stroeven M (2007). Computer simulation study of concrete's self-healing capacity due to unhydrated cement nuclei in interfacial transition zones. Proc $1^{\text {st }}$ Int Conf Self-healing materials. Noordwijk aan Zee, The Netherlands, 51 (abstract), No: 6 (paper on CD).

Hershey AV (1954). The elasticity of an isotropic aggregate of anisotropic cubic crystals. J Appl Mech 21(3):236-40.

Hilfer R (1991). Geometric and dielectric characterization of porous media. Phys Rev B 44:60-75.

Holliday L, Thackray G (1964). Heterogeneity in complex materials and the concept of the representative cell. Nature 201:270-81.

Hu J, Chen H, Stroeven P (2005). Spatial dispersion of aggregate in concrete; a computer simulation study. Comp and Concr 3(5):301-12.

Hu J, Stroeven P (2005). Local porosity analysis of pore structure in cement paste. Cem Concr Res 35(2):233-42.

Keeton JR (1965). Photo-elastic determination of strain distribution in cement pastes, mortars and concretes. Techn Rep R404. Port Hueneme (California): US Naval Civil Engineering Laboratory.

Kendall MG, Moran PAP (1963). Geometric probability. London: C. Griffin \& Co.

Pearson K (1902). Note on Francis Galton's difference problem. Biometrika 1:390-9. 
Pearson K (1924/1931). Tables for statisticians and biometricians. Teddington (England): Cambridge University Press, I (1924) and II (1931).

RILEM TC QFS (2004). Quasi-brittle fracture scaling and size effects- Final Report. Mat Struct 37:547-68.

Stroeven M, Askes H, Sluys LJ (2002). A numerical approach to determine representative volumes for granular materials. Proc World Conf Computational Mechanics, WCCMV, Vienna, paper ID: 80395, available on Internet (http://wccm.tuwien.ac.at).

Stroeven P (1973). Some aspects of the micro-mechanics of concrete. Ph.D. Thesis. Delft: Delft University Press.

Stroeven P (2000). A stereological approach to roughness of fracture surfaces and tortuosity of transport paths in concrete. Cem Concr Comp 22:331-41.

Stroeven P (2003). Implications of the law of aggregation of matter in concrete technology. In: Brandt AM, Li VC, Marshall IH eds. Proceedings Brittle Matrix Composite 7 (Warsaw). Cambridge: Woodhead Publ Ltd., 129-42.

Stroeven P, Guo Z (2006). Modern routes to explore concrete's complex pore space. Image Anal Stereol 25: 75-86.

Stroeven P, Guo Z, He H, Hu J, Stroeven M (2007a). On discrete element packing simulation of concrete aggregate. Proceedings Int Conf Stereol XII. (at: http://ICSXII.univ-st-etienne.fr/)

Stroeven P, Guo Z, Stroeven M (2007b). Does Brazil nut effect influence concrete properties? In: Yeginobali ed. Proceeding $3^{\text {rd }}$ Int. Symp. Sustainability in cement and concrete.): Damla Matbaacilik Reklamcilik ve Yayincilik Tic. Ltd., Ankara, 543-52.

Stroeven P, Sluys LJ, Guo Z, Stroeven M (2006). Virtual reality studies of concrete. Forma 21:227-42

Stroeven P, Stroeven M (2001). Size of representative volume element of concrete assessed by quantitative image analysis and computer simulation. Image Anal Stereol 20(suppl 1):216-20.

Stroeven P, Stroeven M (2003). Dynamic computer simulation of concrete on different levels of the microstructure. Image Anal Stereol 22:1-10 and 91-95.

Tippet LCH (1925). On the extreme individuals and the range of samples taken from a normal population. Biometrika $17: 364-87$.

Tucker J (1927). A study of the compressive strength dispersion of materials with applications. J Frankl Inst 241:751-81.

Underwood EE (1968). Quantitative stereology. Reading: Addison-Wesley Publ Co.

Weibull W (1939). A statistical theory of the strength of materials. In: Proceedings Royal Swedish Inst of Engr Res 151, Stockholm.

Williams SR, Philipse AP (2003). Random packings of spheres and spherocylinders simulated by mechanical contraction. Phys Rev E 67(051301):1-9. 